\title{
Icariin inhibits oxidized low-density lipoprotein-induced proliferation of vascular smooth muscle cells by suppressing activation of extracellular signal-regulated kinase 1/2 and expression of proliferating cell nuclear antigen
}

\author{
YANWU HU ${ }^{1,2}$, KAI LIU ${ }^{1}$, MENGTONG YAN ${ }^{1}$, YANG ZHANG ${ }^{1}$, YADI WANG ${ }^{1}$ and LIQUN REN ${ }^{1}$ \\ ${ }^{1}$ Department of Experimental Pharmacology and Toxicology, School of Pharmaceutical Science, Jilin University, Changchun, \\ Jilin 130021; ${ }^{2}$ School of Pharmaceutical and Food Science, Tonghua Normal University, Tonghua, Jilin 134002, P.R. China
}

Received March 10, 2015; Accepted January 6, 2016

DOI: $10.3892 / \mathrm{mmr} .2016 .4813$

\begin{abstract}
Icariin, a flavonoid isolated from the traditional Chinese herbal medicine Epimedium brevicornum Maxim, has been shown to possess anti-inflammatory, anti-oxidant and anti-atherosclerotic activities in vivo and in vitro. The aim of the present study was to investigate the effects of icariin on oxidized low-density lipoprotein (ox-LDL)-induced proliferation of vascular smooth muscle cells (VSMCs) and the possible underlying mechanism. VSMCs were cultured and pre-treated with various concentrations of icariin $(0,10,20$ or $40 \mu \mathrm{m})$ prior to stimulation by ox-LDL $(50 \mu \mathrm{g} / \mathrm{ml})$. Cell proliferation was evaluated by an MTT assay. Flow cytometry was used to study the influence of icariin on the cell cycle. Proliferating cell nuclear antigen (PCNA) expression and phosphorylation levels of extracellular signal-regulated kinase (ERK)1/2 were detected by western blot analysis. The results indicated that icariin significantly inhibited ox-LDL-induced proliferation of VSMCs and phosphorylation of ERK1/2. Furthermore, icariin also blocked the ox-LDL-induced cell-cycle progression at G1/S-interphase and downregulated the expression of PCNA in VSMCs. In conclusion, the present study indicated for the first time that icariin reduced the amount of ox-LDL-induced proliferation of VSMCs through suppression of PCNA expression and inactivation of ERK1/2.
\end{abstract}

Correspondence to: Professor Liqun Ren, Department of Experimental Pharmacology and Toxicology, School of Pharmaceutical Science, Jilin University, 1266 Fujin Road, Changchun, Jilin 130021, P.R. China

E-mail: renlqjlu@163.com

Key words: icariin, oxidized low-density lipoprotein, vascular smooth muscle cell proliferation, proliferating cell nuclear antigen, extracellular signal-regulated kinase $1 / 2$

\section{Introduction}

Atherosclerosis is a chronic vascular inflammatory disease, characterized by narrowing and rigidity of the lumen as a result of cholesterol and lipid accumulation (1,2). Abnormal proliferation of intimal vascular smooth muscle cells (VSMCs) leads to intimal thickening of the aorta, and has an important role in initiation and amplification of atherogenesis (3). Oxidized low-density lipoprotein (ox-LDL) is a mitogen in VSMCs and stimulates the proliferation of VSMCs and activation of the extracellular signal-regulated protein kinase (ERK)1/2 signaling pathway. Therefore, the ox-LDL-induced proliferation of VSMCs in the intima of the arterial wall is considered to be an important factor in atherosclerotic plaque development (4).

Epimedium brevicornum Maxim, a traditional Chinese herbal medicine, has been widely used for tonifying kidneys and strengthening bone for thousands of years in China, Korea and Japan (5-7). Icariin $\left(\mathrm{C}_{33} \mathrm{H}_{40} \mathrm{O}_{15}\right.$; molecular weight, 676.67; Fig. 1), a flavonoid isolated from Epimedium brevicornum Maxim, is considered as the main pharmacological active constituent $(8,9)$ and has been reported to possess various pharmacological effects, including anti-inflammatory, anti-osteoporosis (10), anti-tumor (11), immunoregulatory (12) and anti-oxidative actions (13). In addition, icariin has been shown to have beneficial effects on cardiovascular diseases such as atherosclerosis $(14,15)$. However, the potential mechanisms of action of icariin against atherosclerosis have remained to be fully elucidated. In view of this, the present study was designed to elucidate whether icariin can attenuate the initiation and progression of atherosclerosis. The effects of icariin on ox-LDL-induced proliferation of VSMCs were assessed, and the results indicated that they are mediated via suppression of cell-cycle regulatory protein proliferating cell nuclear antigen (PCNA) and deactivation of ERK1/2.

\section{Materials and methods}

Cell culture. Human aortic vascular smooth muscle cells (HA-VSMCs) were obtained from the Chinese Academy of Sciences Cell Bank (Shanghai, China) and cultured in 100-mm 
dishes in Dulbecco's modified Eagle's medium (Gibco; Thermo Fisher Scientific, Inc., Waltham, MA, USA) supplemented with $10 \%$ heat-inactivated fetal bovine serum (FBS; Sijiqing Bioengineering Material Co., Ltd. Hangzhou, China) and $1 \%$ penicillin/streptomycin (Sigma-Aldrich, St Louis, MO, USA) at $37^{\circ} \mathrm{C}$ in a humidified atmosphere containing $5 \% \mathrm{CO}_{2}$.

Cell viability assay. VSMCs in the logarithmic growth phase were seeded into 96-well plates at a density of $1 \times 10^{4}$ cells per well and incubated for $24 \mathrm{~h}$ at $37^{\circ} \mathrm{C}$ in an atmosphere containing $5 \% \mathrm{CO}_{2}$. After pre-treatment with the indicated concentrations of icariin (purity, >98\%; Vic's Biological Technology Co., Ltd, Sichuan, China; $0,10,20$ or $40 \mu \mathrm{m}$ ) for $24 \mathrm{~h}$ prior to stimulation with oxidized low-density lipoprotein (ox-LDL; Yiyuan Biotechnologies Co., Ltd, Guangzhou, China; $50 \mu \mathrm{g} / \mathrm{ml}$ ) for the indicated times ( 24 or $48 \mathrm{~h}$ ). Subsequently, the medium was discarded and 3-(4,5-dimethylthiazol-2-yl)-2,5-diphenyltetrazolium bromide (MTT; Sigma-Aldrich) solution was added at a final concentration of $0.5 \mathrm{mg} / \mathrm{ml}$, followed by incubation for $4 \mathrm{~h}$ at $37^{\circ} \mathrm{C}$. The MTT solution was carefully removed and $150 \mu$ l dimethyl sulfoxide (Sigma-Aldrich) was added to each well followed by a 15-min incubation. The absorbance of each well was measured using a microplate reader (Multiskan MK3; Thermo Fisher Scientific, Inc.) with a reference wave length of $490 \mathrm{~nm}$. The initial absorbance at $0 \mathrm{~h}$, prior to icariin treatment, was also measured.

Cell cycle analysis. VSMCs in the logarithmic growth phase were seeded into six-well plates at a density of $1 \times 10^{4}$ cells per well and then incubated for $24 \mathrm{~h}$ at $37^{\circ} \mathrm{C}$ in an atmosphere containing $5 \% \mathrm{CO}_{2}$. After pre-treatment with the indicated concentrations of icariin $(0,10,20$ or $40 \mu \mathrm{m})$ for $24 \mathrm{~h}$, cells were incubated with or without ox-LDL $(50 \mu \mathrm{g} / \mathrm{ml})$ for a further $24 \mathrm{~h}$. The cells were trypsinized, collected and washed twice with ice-cold phosphate-buffered saline (PBS) prior to fixing in $70 \%$ cold ethanol at $4^{\circ} \mathrm{C}$ overnight. Next, the fixed cells were re-suspended in PBS containing $100 \mu \mathrm{g} / \mathrm{ml}$ RNase A (Sigma-Aldrich) and $50 \mu \mathrm{g} / \mathrm{ml}$ propidium iodide (PI; Sigma-Aldrich) for $30 \mathrm{~min}$ at room temperature. Cells were then analyzed using a FACSCalibur flow cytometer (Becton Dickinson, San Jose, CA, USA). The percentages of cells in G0/G1, S and G2/M phases were determined using ModFit LT V3.3.11 software (Verity Software House Inc., Topsham, ME, USA).

Western blot analysis. VSMCs in the logarithmic growth phase were seeded into six-well plates for incubation for $24 \mathrm{~h}$ at $37^{\circ} \mathrm{C}$ in an atmosphere containing $5 \% \mathrm{CO}_{2}$. After pre-treatment with the indicated concentrations of icariin $(0$, 10,20 or $40 \mu \mathrm{m}$ ) for $24 \mathrm{~h}$, the cells were incubated with or without ox-LDL $(50 \mu \mathrm{g} / \mathrm{ml})$ for another $24 \mathrm{~h}$. Subsequently, VSMCs were scraped in ice-cold PBS and lysed in cold lysis buffer (Beyotime Institute of Biotechnology, Haimen, China). After centrifugation at $13,000 \mathrm{x}$ g, the supernatant (total protein extract) was separated and quantified using a bicinchoninic acid protein assay kit (Thermo Fisher Scientific, Inc.). Equal amounts of protein samples $(50 \mu \mathrm{g})$ were loaded onto 10\% SDS-PAGE gels (Applygen Technologies, Inc., Beijing, China) and transferred to nitrocellulose membranes (EMD Millipore, Billerica, MA, USA). The membranes

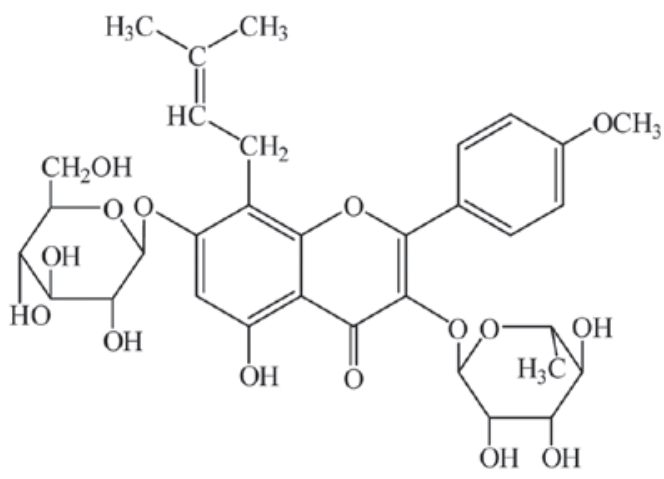

Figure 1. Chemical structure of icariin $\left(\mathrm{C}_{33} \mathrm{H}_{40} \mathrm{O}_{15}\right.$; molecular weight, 676.67).

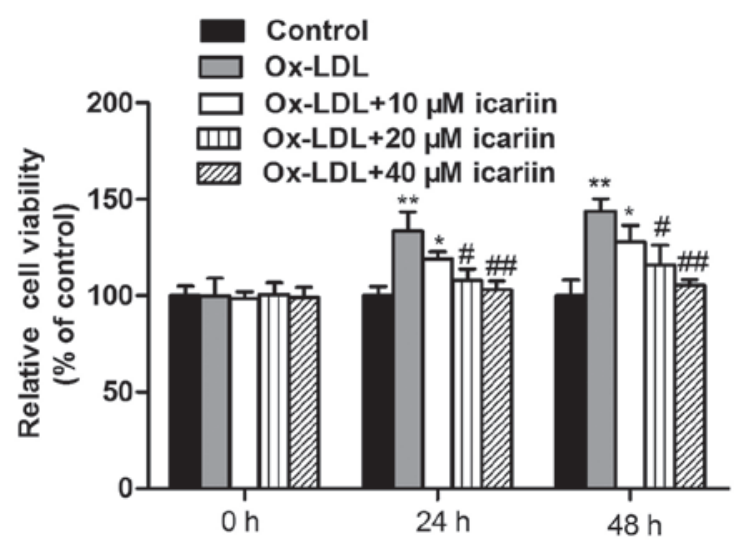

Figure 2. Effects of icariin on ox-LDL-induced proliferation of VSMCs VSMCs were pre-treated for $24 \mathrm{~h}$ with different concentrations of icariin and then exposed to ox-LDL $(50 \mu \mathrm{g} / \mathrm{ml})$ for 24 or $48 \mathrm{~h}$. Cell viability was assessed by the MTT method. Icariin pre-treatment significantly increased the cell viability. Values are expressed as the mean \pm standard deviation from three independent experiments. ${ }^{*} \mathrm{P}<0.05$ and ${ }^{* *} \mathrm{P}<0.01$ vs. the control group, ${ }^{\#} \mathrm{P}<0.05$ and $^{\# \#} \mathrm{P}<0.01$ vs. the ox-LDL-simulated group. VSMC, vascular smooth muscle cell; ox-LDL, oxidized low-density lipoprotein.

were blocked with $5 \%$ fat-free milk in Tris-buffered saline containing $0.05 \%$ Tween 20 (TBST; Zhongtian Jingwei Technologies, Inc., Beijing, China) and incubated with the primary rabbit anti-human monoclonal antibodies against ERK1/2 (dilution, 1:1,000; Abcam, Cambridge, MA, USA; cat. no. ab36911), anti-phosphorylated-ERK1/2 (dilution, 1:1,000; Abcam; cat. no. 50011) or anti-PCNA (dilution, 1:1,000; Abcam; cat. no. ab92552) or anti-GAPDH (dilution, 1:1,000; Sigma-Aldrich; cat. no. sab4300645) at $4^{\circ} \mathrm{C}$ overnight. Following incubation, the membranes were washed three times in TBST for $15 \mathrm{~min}$ and the membranes were incubated with horseradish-peroxidase-labeled goat anti-rabbit secondary antibody for $1 \mathrm{~h}$ at room temperature (dilution, 1:5,000; Santa Cruz Biotechnology, Inc., Dallas, TX, USA; cat. no. sc-45101). Following three further washes in TBST, the protein expression levels were visualized using the enhanced chemiluminescence kit, BeyoECL Plus (Beyotime Institute of Biotechnology), images of the blots were captured on X-ray films (GE Healthcare, Little Chalfont, UK) and were analyzed using Image J version 1.46 (National Institutes of Health, Bethesda, MD, USA). GAPDH was used as the protein loading control. 

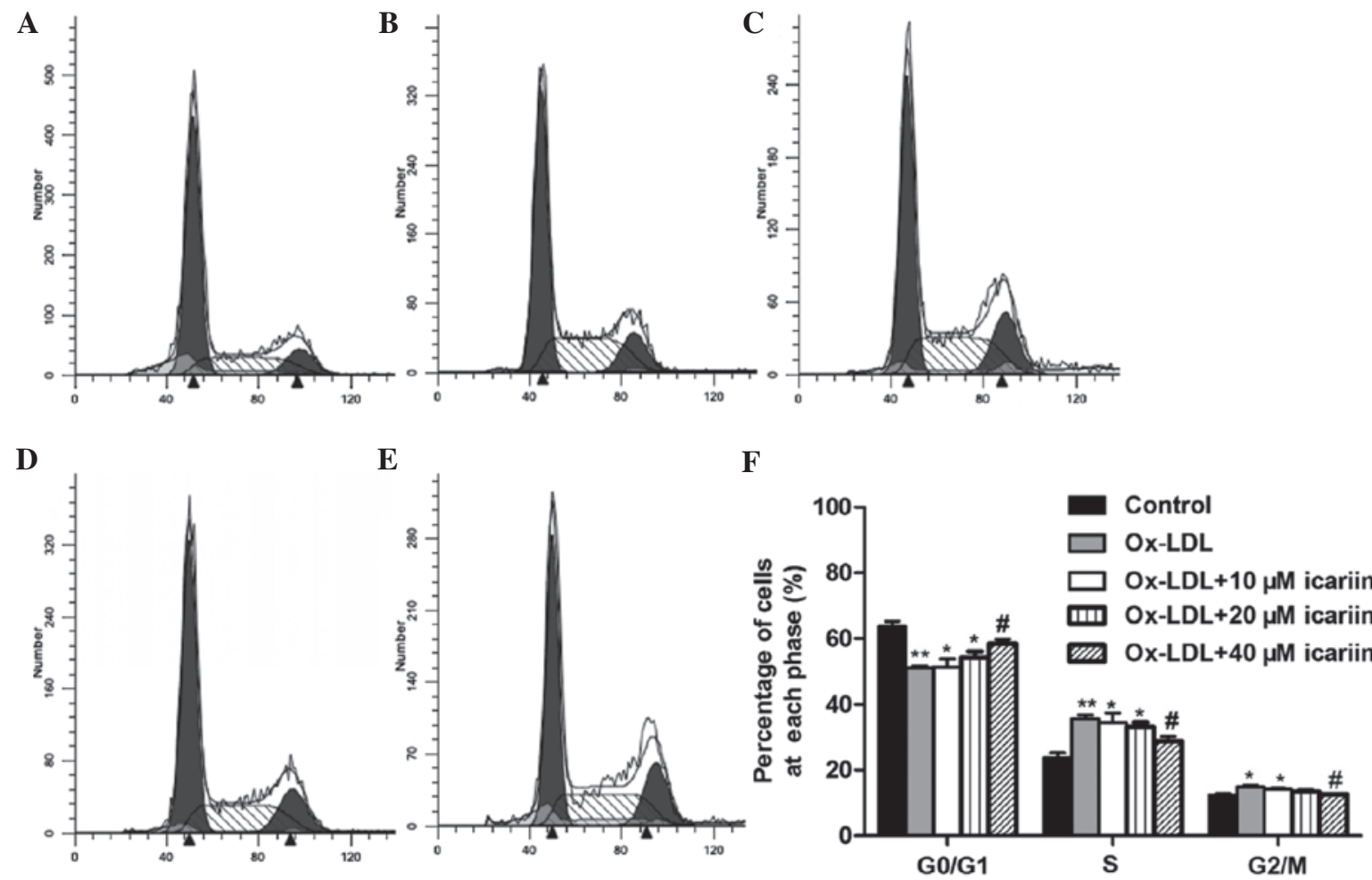

Figure 3. Effects of icariin on the cell cycle of VSMCs induced by ox-LDL. VSMCs were pre-treated with various concentrations of icariin $(0,10,20,40 \mu \mathrm{m})$ and then incubated with ox-LDL $(50 \mu \mathrm{g} / \mathrm{ml})$ for another $24 \mathrm{~h}$. The cell cycle distribution was determined by assessing the individual nuclear DNA content reflected by the fluorescence intensity of incorporated propidium iodide. Flow cytometric analysis of (A) VSMCs without any treatment (control), (B) VSMCs induced by ox-LDL and (C-E) VSMCs induced by ox-LDL following pre-treatment with icariin at 10, 20 or $40 \mu \mathrm{m}$, respectively. (F) Percentages of cells in G0/G1, S and G2/M phases after the indicated treatments. Representative flow cytometry graphs are shown and results are expressed as the mean \pm standard deviation from three independent experiments. ${ }^{*} \mathrm{P}<0.05$ and ${ }^{* * *} \mathrm{P}<0.01$ vs. the control group, ${ }^{*} \mathrm{P}<0.05$ vs. the ox-LDL-simulated group. VSMC, vascular smooth muscle cell; ox-LDL, oxidized low-density lipoprotein.

Statistical analysis. Statistical analysis was performed using the SPSS 17.0 statistical package (SPSS, Inc., Chicago, IL, USA). Values are expressed as the mean \pm standard deviation. One-way analysis of variance was applied for multiple comparisons and the least significant difference test was applied for intra-group comparison. $\mathrm{P}<0.05$ was considered to indicate a statistically significant difference.

\section{Results}

Icariin inhibits ox-LDL-induced VSMC proliferation. To evaluate the effects of icariin on the proliferation of VSMCs induced by ox-LDL, the cell viability was assessed using an MTT assay. As shown in Fig. 2, exposure of the cells to ox-LDL for 24 or $48 \mathrm{~h}$ significantly increased the cell viability compared with that in the control group. However, icariin inhibited ox-LDL-induced VSMC proliferation in a concentration-dependent manner.

Icariin reduces ox-LDL-induced cell cycle progression and PCNA expression. To clarify the effect of icariin on cell-cycle regulation, the cell cycle distribution of ox-LDL-induced VSMCs was assessed using flow cytometry. As shown in Fig. 3, treatment with ox-LDL markedly increased the percentage of VSMCs in $\mathrm{S}$ and G2/M phases and correspondingly decreased the percentage of cells in G0/G1 phase. However, pre-treatment with icariin significantly reversed these effects in a concentration-dependent manner. To assess whether the effects of icariin on the cell cycle were associated with the expression of PCNA, western blot analysis was performed. As shown in Fig. 4, the protein expression of PCNA was markedly increased following ox-LDL treatment, which was inhibited by icariin in a dose-dependent manner. The western blot results were in line with the findings of the cell cycle analysis, as ox-LDL enhanced the population of cells in S phase, which was accompanied an increased expression of PCNA, while icariin pre-treatment was able to inhibit these effects in a concentration-dependent manner. These results suggested that icariin inhibits ox-LDL-induced VSMC proliferation by blocking cell cycle progression.

Icariin inhibits ox-LDL-induced phosphorylation of ERK1/2. To demonstrate whether icariin inhibited ox-LDL-induced VSMC proliferation by inhibiting the activation of ERK1/2, the phosphorylation levels of ERK1/2 were examined. As shown in Fig. 5, ox-LDL and icariin had no effect on the levels of total ERK1/2. VSMCs incubated with ox-LDL for $24 \mathrm{~h}$ showed markedly enhanced ERK1/2 phosphorylation, which was significantly and dose-dependently inhibited by icariin pre-treatment. These results suggested that icariin may reduce ox-LDL-induced proliferation of VSMCs, at least in part, via inhibition of ox-LDL-induced ERK1/2 phosphorylation.

\section{Discussion}

Atherosclerosis is a chronic vascular inflammatory disease (16). It is characterized by the formation of atherosclerotic plaques 


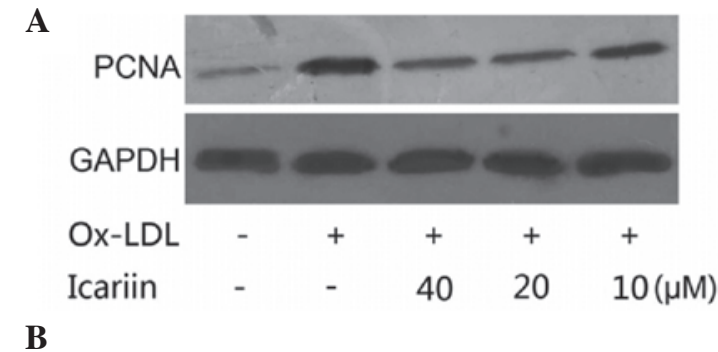

$\mathbf{B}$

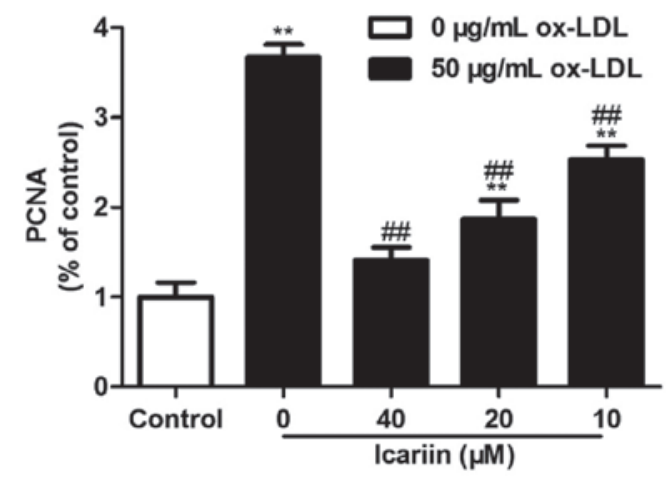

Figure 4. Effects of icariin on ox-LDL-induced PCNA expression in VSMCs. VSMCs were pre-treated with various concentrations of icariin $(0,10,20$ or $40 \mu \mathrm{m})$, and then incubated with ox-LDL $(50 \mu \mathrm{g} / \mathrm{ml})$ for another $24 \mathrm{~h}$. Proteins were extracted from the cells and assessed by western blot analysis. GAPDH served as the loading control. (A) Western blot assay for PCNA expression. Blots are representative of three independent experiments. (B) PCNA expression was quantified by densitometric analysis and shown as the ratio of the control. Values are expressed as the mean \pm standard deviation from three independent experiments. ${ }^{* *} \mathrm{P}<0.01$ vs. the control group, ${ }^{\# /} \mathrm{P}<0.01$ vs. the ox-LDL-simulated group. VSMC, vascular smooth muscle cell; ox-LDL, oxidized low-density lipoprotein; PCNA, proliferating cell nuclear antigen; GAPDH, glyceraldehyde 3-phosphate dehydrogenase.

consisting of foam cells, leukocytes, platelets, inflamed smooth muscle cells and endothelial cells (17). It is increasingly recognized that ox-LDL has a critical role in the promotion of atherosclerosis initiation, progression and plaque destabilization (18). Several studies have indicated that ox-LDL can stimulate the proliferation of VSMCs and the activation of ERKs $(4,19)$. Therefore, VSMC proliferation induced by ox-LDL in the intima of the arterial wall is thought to have a critical role in the development of atherosclerotic lesions (4). However, inhibition of VSMC proliferation represents a potentially important therapeutic strategy for the prevention and treatment of atherosclerosis (20). The present study showed that ox-LDL induced VSMC proliferation and provided the first evidence that icariin inhibited ox-LDL-stimulated VSMC proliferation in a concentration-dependent manner.

The cell cycle is a highly regulated process that involves a complex cascade of events to regulate cell proliferation (21-23). It comprises three distinct phases: The G0/G1 phase, the DNA synthesis-associated $\mathrm{S}$ phase and the $\mathrm{G} 2 / \mathrm{M}$ phase $(23,24)$. Under normal conditions, VSMCs proliferate at low rates, largely remaining in the G0/G1 phase of the cell cycle. Following proliferative stimulation, VSMCs re-enter the cell cycle $(3,25)$. In the present study, flow-cytometric analysis indicated that ox-LDL treatment promoted the proliferation of VSMCs and increased the S-phase population with a simultaneous decrease in the G0/G1-phase population, while pre-treatment with icariin significantly reversed these effects.
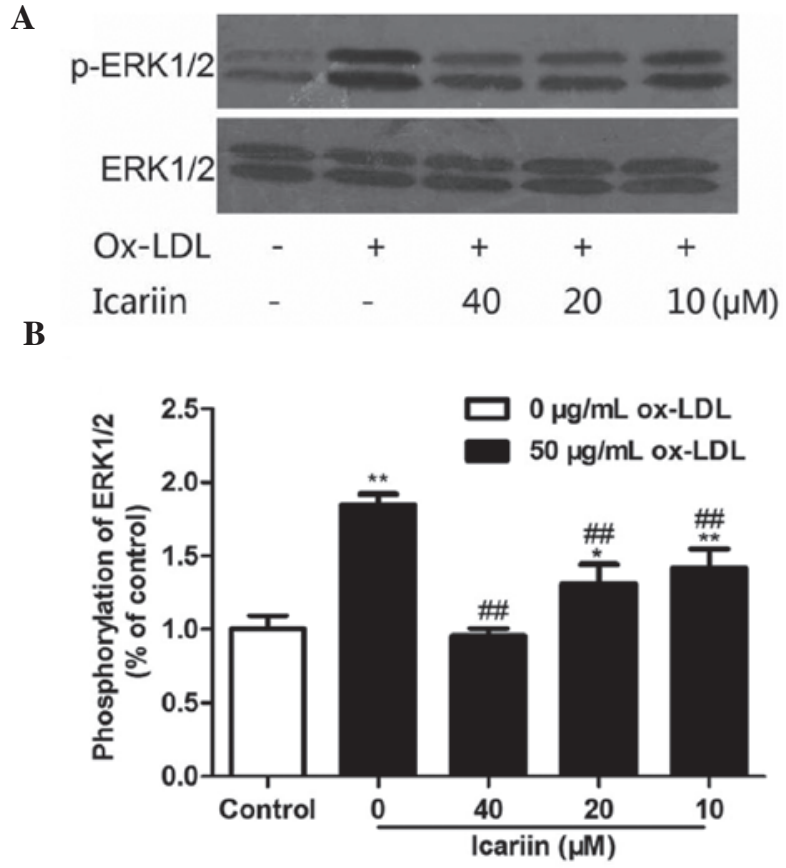

Figure 5. Effects of icariin on ERK1/2 phosphorylation in VSMCs. VSMCs were pre-treated with various concentrations of icariin $(0,10,20$ or $40 \mu \mathrm{m})$ and then exposed to ox-LDL $(50 \mu \mathrm{g} / \mathrm{ml})$ for another $24 \mathrm{~h}$. Proteins were extracted from the cells and assessed by western blot analysis. Total ERK1/2 served as a loading control. (A) Western blot assays for ERK1/2 and p-ERK1/2 expression. Blots are representative of three independent experiments. (B) The p-ERK1/2/ERK1/2 expression ratio was quantified by densitometric analysis and shown as the ratio of the control. Values are expressed as the mean \pm standard deviation from three independent experiments. ${ }^{*} \mathrm{P}<0.05$ and ${ }^{* *} \mathrm{P}<0.01$ vs. the control group, ${ }^{\#} \mathrm{P}<0.01$ vs. the ox-LDL-simulated group. VSMC, vascular smooth muscle cell; ox-LDL, oxidized low-density lipoprotein; p-ERK, phosphorylated extracellular signal-regulated kinase.

The results suggested that icariin may reduce ox-LDL-induced proliferation of VSMCs by inhibiting their transition from G0/G1 phase to $S$ phase.

PCNA is involved in a number of essential cellular processes, including DNA repair, DNA replication and cell-cycle regulation (26), and is regulated by a variety of mechanisms that act at the transcriptional as well as the post-transcriptional level (27). PCNA is required for G0/G1-to-S phase transition and its synthesis is tightly regulated during the cell cycle (4). The present study found that the percentage of VSMCs in $\mathrm{S}$ and $\mathrm{G} 2 / \mathrm{M}$ phase increased after treatment with ox-LDL, which was inhibited by pre-treatment with icariin. These findings were in line with the effects of ox-LDL and icariin on the protein expression of PCNA: Ox-LDL treatment enhanced the expression of PCNA, while icariin dose-dependently inhibited these increases. It is therefore likely that icariin inhibited ox-LDL-induced VSMC proliferation through inhibition of PCNA expression.

ERK is a widely expressed protein kinase and an intracellular signaling molecule that is involved in cell proliferation (28). Previous studies have indicated that ox-LDL induces VSMC proliferation through activation of the ERK pathway $(19,29,30)$. In line with these results, the present study revealed that the phosphorylation of ERK1/2 in VSMCs was enhanced by ox-LDL, which was inhibited by pre-treatment with icariin. These results suggested that icariin significantly 
inhibited ox-LDL-induced proliferation of VSMCs by blocking cell-cycle progression, partly via inhibiting the ox-LDL-induced activation of ERK1/2.

In conclusion, the present study demonstrated that icariin inhibited the proliferation of VSMCs stimulated by ox-LDL via decreasing the $S$-phase population of the cell cycle. Ox-LDL-induced phosphorylation of ERK1/2 and the expression of PCNA were also suppressed by icariin. These findings suggested that icariin may inhibit ox-LDL-induced proliferation of VSMCs by inactivating the ERK1/2 signaling pathway and by suppressing the expression of PCNA. Icariin may therefore be able to reduce the development of atherosclerosis.

\section{Acknowledgements}

The present study was supported by the Jilin Natural Science Foundation (grant no. 20150101221JC) and by the Applied Research Project of Tonghua Normal University (grant no. 2014096).

\section{References}

1. Dell'omo G, Penno G, Pucci L, Lucchesi D, Fotino C, Del Prato S and Pedrinelli R: ACE gene insertion/deletion polymorphism modulates capillary permeability in hypertension. Clin Sci (Lond) 111: 357-364, 2006.

2. Zhu F, Li C, Jin XP, Weng SX, Fan LL, Zheng Z, Li WL, Wang F, Wang WF, Hu XF, et al: Celastrol may have an anti-atherosclerosis effect in a rabbit experimental carotid atherosclerosis model. Int J Clin Exp Med 7: 1684-1691, 2014.

3. Boehm M and Nabel EG: Cell cycle and cell migration: New pieces to the puzzle. Circulation 103: 2879-2881, 2001.

4. Chang WC, Yu YM, Chiang SY and Tseng CY: Ellagic acid suppresses oxidised low-density lipoprotein-induced aortic smooth muscle cell proliferation: Studies on the activation of extracellular signal-regulated kinase $1 / 2$ and proliferating cell nuclear antigen expression. Br J Nutr 99: 709-714, 2008.

5. Xie F, Wu CF, Lai WP, Yang XJ, Cheung PY, Yao XS, Leung PC and Wong MS: The osteoprotective effect of Herba epimedii (HEP) extract in vivo and in vitro. Evid Based Complement Alternat Med 2: 353-361, 2005.

6. Hidaka S, Okamoto Y, Yamada Y, Kon Y and Kimura T: A Japanese herbal medicine, Chujo-to, has a beneficial effect on osteoporosis in rats. Phytother Res 13: 14-19, 1999.

7. Sakamoto S, Sassa S, Kudo H, Suzuki S, Mitamura T and Shinoda H: Preventive effects of a herbal medicine on bone loss in rats treated with a GnRH agonist. Eur J Endocrinol 143: $139-142,2000$

8. Xiao-Hong D, Chang-Qin X, Jian-Hua H, Wen-Jiang Z and Bing S: Icariin delays homocysteine-induced endothelial cellular senescence involving activation of the PI3K/AKT-eNOS signaling pathway. Pharm Biol 51: 433-440, 2013.

9. Zeng KW, Fu H, Liu GX and Wang XM: Icariin attenuates lipopolysaccharide-induced microglial activation and resultant death of neurons by inhibiting TAK1/IKK/NF-kappaB and JNK/p38 MAPK pathways. Int Immunopharmacol 10: 668-678, 2010.

10. Zhang G, Qin L, Sheng H, Yeung KW, Yeung HY, Cheung WH, Griffith J, Chan CW, Lee KM and Leung KS: Epimedium-derived phytoestrogen exert beneficial effect on preventing steroid-associated osteonecrosis in rabbits with inhibition of both thrombosis and lipid-deposition. Bone 40: 685-692, 2007.

11. Zhang DC, Liu JL, Ding YB, Xia JG and Chen GY: Icariin potentiates the antitumor activity of gemcitabine in gallbladder cancer by suppressing NF-кB. Acta Pharmacol Sin 34: 301-308, 2013.
12. Yap SP, Shen P, Li J, Lee LS and Yong EL: Molecular and pharmacodynamic properties of estrogenic extracts from the traditional Chinese medicinal herb, Epimedium. J Ethnopharmacol 113: 218-224, 2007.

13. Chen Y, Huang JH, Ning Y and Shen ZY: Icariin and its pharmaceutical efficacy: Research progress of molecular mechanism. J Chin Integr Med 9: 1179-1184, 2011 (In Chinese).

14. Xu CQ, Liu BJ, Wu JF, Xu YC, Duan XH, Cao YX and Dong JC: Icariin attenuates LPS-induced acute inflammatory responses: Involvement of PI3K/Akt and NF-kappaB signaling pathway. Eur J Pharmacol 642: 146-153, 2010.

15. Xu HB and Huang ZQ: Vasorelaxant effects of icariin on isolated canine coronary artery. J Cardiovasc Pharmacol 49: 207-213, 2007.

16. Nurgeldiyeva MJ, Hojakuliyev BG and Muhammedov MB: Correlation of atherogenesis with an infection of Candida albicans. Int J Clin Exp Med 7: 2137-2143, 2014.

17. Butcher MJ, Herre M, Ley K and Galkina E: Flow cytometry analysis of immune cells within murine aortas. J Vis Exp: pii: 2848, 2011.

18. Li Q, Wang Y, Li H, Shen G and Hu S: Ox-LDL influences peripheral Th17/Treg balance by modulating Treg apoptosis and Th17 proliferation in atherosclerotic cerebral infarction. Cell Physiol Biochem 33: 1849-1862, 2014.

19. Yang CM, Chien CS, Hsiao LD, Pan SL, Wang CC, Chiu CT and Lin CC: Mitogenic effect of oxidized low-density lipoprotein on vascular smooth muscle cells mediated by activation of Ras/Raf/MEK/MAPK pathway. Br J Pharmacol 132: 1531-1541, 2001.

20. Abate-Daga D, Hanada K, Davis JL, Yang JC, Rosenberg SA and Morgan RA: Expression profiling of TCR-engineered T cells demonstrates overexpression of multiple inhibitory receptors in persisting lymphocytes. Blood 122: 1399-1410, 2013.

21. Tian $\mathrm{R}, \mathrm{Li}$ Y and Gao M: Shikonin causes cell cycle arrest and induces apoptosis by regulating the EGFR/NF- $\mathrm{BB}$ signaling pathway in human epidermoid carcinoma A431 cells. Biosci Rep 35: pii: e00189, 2015.

22. Li QY, Zhu YF, Zhang M, Chen L, Zhang Z, Du YL, Ren GQ, Tang JM, Zhong MK and Shi XJ: Chlorogenic acid inhibits hypoxia-induced pulmonary artery smooth muscle cells proliferation via c-Src and Shc/Grb2/ERK2 signaling pathway. Eur J Pharmacol 751: 81-88, 2015.

23. Lu W, Du S and Wang J: Berberine inhibits the proliferation of prostate cancer cells and induces $\mathrm{G}_{0} / \mathrm{G}_{1}$ or $\mathrm{G}_{2} / \mathrm{M}$ phase arrest at different concentrations. Mol Med Rep 11: 3920-3924: 2015.

24. Elledge SJ: Cell cycle checkpoints: Preventing an identity crisis. Science 274: 1664-1672, 1996.

25. Kim JY, Kim KH, Lee WR, An HJ, Lee SJ, Han SM, Lee KG, Park YY, Kim KS and Lee YS: Apamin inhibits PDGF-BB-induced vascular smooth muscle cell proliferation and migration through suppressions of activated Akt and Erk signaling pathway. Vascul Pharmacol 70: 8-14, 2015.

26. He H, Tan CK, Downey KM and So AG: A tumor necrosis factor alpha- and interleukin 6-inducible protein that interacts with the small subunit of DNA polymerase delta and proliferating cell nuclear antigen. Proc Natl Acad Sci USA 98: 11979-11984, 2001.

27. Li YY, Wang L and Lu CD: An E2F site in the 5'-promoter region contributes to serum-dependent up-regulation of the human proliferating cell nuclear antigen gene. Febs Lett 544: 112-118, 2003.

28. Roskoski R Jr: ERK1/2 MAP kinases: Structure, function and regulation. Pharmacol Res 66: 105-143, 2012.

29. Zhang Z, Zhang M, Li Y, Liu S, Ping S, Wang J, Ning F, Xie F and $\mathrm{Li} \mathrm{C}$ : Simvastatin inhibits the additive activation of ERK1/2 and proliferation of rat vascular smooth muscle cells induced by combined mechanical stress and oxLDL through LOX-1 pathway. Cell Signal 25: 332-340: 2013.

30. Farrokhi E, Samani KG and Chaleshtori MH: Oxidized low-density lipoprotein increases bone sialoprotein expression in vascular smooth muscle cells via runt-related transcription factor 2. Cell Signal 349: 240-243, 2015. 